\title{
ORIENTAÇÃO EMPREENDEDORA INDIVIDUAL SOB MEDIDA: DESENVOLVIMENTO DE ESCALA VOLTADA AO EMPREENDEDORISMO SOCIAL
}

Márcia Maria Garçon ${ }^{1}$

Vânia Maria Jorge Nassif ${ }^{2}$

\footnotetext{
${ }^{1}$ Pós-doutorado/ Programa de Pós-Graduação em Administração / Universidade Nove de Julho

${ }^{2}$ Mestrado e Doutorado / Programa de Pós-Graduação em Administração / Universidade Nove de Julho
} 


\section{ORIENTAÇÃO EMPREENDEDORA INDIVIDUALSOB MEDIDA: DESENVOLVIMENTO DE ESCALA VOLTADA AO EMPREENDEDORISMO SOCIAL}

Resumo: Este artigo apresenta a validação de conteúdo de itens de uma escala que pretende avaliar e mensurar a OEI voltada para o empreendedorismo social - OEI-Social. Parte-se da proposição que o empreendedorismo social estabelece uma ligação direta com valores sociais que influenciam a proatividade, a inovação e a tomada de risco para empreender em negócios sociais. Assim, requer um instrumento específico para sua avaliação, construído pela abordagem integrativa das teorias que tratam o afetivo e o cognitivo no comportamento empreendedor. O estudo exploratório segue a metodologia proposta por Churchill (1979), DeVellis (2003) e Johnson \& Morgan (2016). Participaram dessa validação, 24 doutores em Empreendedorismo que avaliaram 24 itens construídos a partir da literatura. Dezoito foram validados em três dimensões: Proatividade Social, Inovação Social e Tomada de Risco Social em apenas uma rodada. Propõe ainda uma quarta dimensão ao instrumento: Mudança Social, que representa o comportamento pró-social dos empreendedores.

Palavras-chave: Orientação Empreendedora Individual. Empreendedorismo Social. Escala.

\section{$1 \quad$ Introdução}

Para além das políticas de responsabilidade social corporativa e sustentabilidade das organizações tradicionais, tem-se observado um sólido crescimento de iniciativas empresariais criadas com o objetivo de alinhar o combate à pobreza e a exclusão social e o respeito ao meio ambiente às práticas do negócio, o chamado empreendedorismo social. Tal fenômeno vem chamando a atenção de pesquisadores que concordam em consubstanciar o empreendedorismo social como uma ação que busca a solução de problemas sociais e ambientais, a partir de formas alternativas de produção econômica podendo estar associada ou não à participação social e democrática (Godói-de-Souza, 2010). Sob esse prisma, a definição mais abrangente de empreendedorismo social pode ser encontrada em Austin, Stevenson \& Wei-Skillern (2012), que afirmam ser uma atividade criadora e inovadora de valor social, que atuam com inovações de metodologia, serviços ou produtos a fim de impulsionar para transformação social. Neste sentido, tais práticas podem ser encontradas tanto em organizações sem fins lucrativos como também nas comerciais ou governamentais. A motivação primeira desses empreendimentos é a criação de valor social em vez de riqueza pessoal (Zadek \& Thake, 1997) e seu driver central é o problema social-ambiental que está sendo abordado.

A forma organizativa do empreendimento social não é única e pode ser definida pelo formato mais eficaz para mobilizar os recursos necessários ao enfrentamento dos problemas sociais (Quintão, 2004; Godói-de-Souza \& Fischer, 2012). Desta maneira, existem iniciativas como os empreendimentos solidários, mais próximos à literatura da Economia Solidária e que utilizam as abordagens normativas do Terceiro Setor e os Negócios Sociais, empresas privadas e com fins lucrativos, que operam sobre os mecanismos de mercado, mas que têm como atividade principal soluções para problemas sociais (Yunus, Moingeon \& Lehmann- 
Ortega, 2010). Neste último, todo lucro deve ser reinvestido no próprio negócio, mas o investidor pode pegar de volta seu dinheiro investido.

Outras classificações mais específicas são encontradas como: a) Negócio com Impacto Social, uma organização que combina as competências de gestão do setor privado com os conhecimentos de gestão social do Terceiro Setor (Barki et al., 2015); b) Empresa Social, aquela que utiliza mecanismos de mercado com a finalidade de minimizar as desigualdades socioeconômicas e o lucro é reinvestido no próprio negócio ou em benefícios para a comunidade (Reficco, Gutiérrez, \& Trujillo, 2006; Borzaga, Depedri \& Galera, 2012); c) Negócio Inclusivo, quando se está focado em gerar emprego e renda para as pessoas pobres que vivem em áreas de risco social e ambiental (Teodósio \& Comini, 2012; Godói-de-Sousa \& Fischer,2012) e d) Negócios da Base da Pirâmide, modelo que ajuda a população de baixa renda a melhorar sua qualidade de vida, fornecendo produtos padrões culturais de cada local e que sejam, ao mesmo tempo, economicamente rentáveis e responsáveis com o meio ambiente (Prahalad, 2005).

A revisão teórica sobre o tema indica que o empreendedorismo social carrega um alto potencial de transformação social, ambiental e econômica e, por isso mesmo, vem atraindo um novo perfil de empreendedores: aqueles que buscam alinhar satisfação profissional aos seus valores pessoais (Barki et al., 2013). Essa atuação empreendedora procura o progresso da sociedade por meio da inclusão social e econômica e procuram mitigar os efeitos secundários indesejáveis da atividade econômica (Social Enterprise Knowledge Network, 2006).

Nesta trilha, esse artigo apresenta um estudo exploratório sobre a orientação empreendedora individual voltada para o social (OEI-Social) que tem o objetivo de construir uma escala de avaliação e mensuração da OEI-Social. Parte-se da proposição teórica que o empreendedorismo social está altamente relacionado à valores sociais e ambientais e, por conta disso, o comportamento do empreendedor social deve ser avaliado e mensurado por um instrumento específico. A escala mais citada na literatura para a avaliação da Orientação Empreendedora Individual é de Bolton \& Lane (2012) que foi construída sob inspiração do construto organizacional de Orientação Empreendedora (OE) e suas dimensões (Miller,1983) e do instrumento empírico de OE proposto por Covin e Slevin (1991).

A escala proposta considera os itens de aspectos cognitivos consagrados na literatura do empreendedorismo adicionando a influência da afetividade e a especificidade do ambiente social. A seguir, apresentamos o estudo em quatro partes: a primeira, a revisão bibliográfica que fundamentou a escala de OEI-Social; a segunda, a metodologia utilizada para a construção e validação dos itens da escala de OEI-Social e a inclusão da escala World Change como quarta dimensão da OEI-Social; a terceira parte traz as discussões e, por fim, as considerações finais sobre as descobertas e os avanços necessários à constituição da escala.

\section{$2 \quad$ Referencial Teórico}

\subsection{Orientação Empreendedora Individual}

O constructo orientação empreendedora (OE) nasce como uma prática do empreendedorismo no contexto das organizações. Ao associar o desempenho organizacional à orientação empreendedora, pioneiramente Miller (1983) destaca que a organização conquista 
um desempenho superior quando guiado por três dimensões, quais sejam, inovação, proatividade e propensão ao risco, caracterizando assim, a OE.

Observando a evolução dos estudos, outros autores (Miller, 1983; Covin \& Slevin, 1991; Zahra, 1993; Zahra \& Covin, 1995; Wiklund \& Shepherd, 2005) corroboram este pressuposto, sugerindo que a $\mathrm{OE}$ pode influenciar positivamente o desempenho da organização ao descobrir novas oportunidades que propiciam conquistas para diferenciar e criar vantagem competitiva. Lumpkin e Dess (1996) ampliam os pressupostos de Miller propondo mais duas dimensões, autonomia e agressividade competitiva, constituindo assim, um framework conceitual de $\mathrm{OE}$ com cinco dimensões, cujos fatores organizacionais e ambientais são aqueles capazes de influenciar a performance da organização.

Outros estudos passaram a empregar a orientação empreendedora no nível individual (Bolton, \& Lane, 2012; DeGennaro, Wright, \& Panza, 2016). Características e atitude empreendedora então são consideradas como aquelas que estimulam o desenvolvimento do empreendedorismo por meio das pessoas. Kollmann, Christofor, \& Kuckertz (2007), Bolton, \& Lane (2012) e Padilla-Meléndez, Fernández-Gámez, \& Molina-Gómez (2014) levantam conjecturas que sob essa perspectiva é possível identificar o porquê de alguns indivíduos descobrirem e explorarem oportunidades em direção a um empreendimento enquanto outros não o fazem. Pelo fato da orientação empreendedora individual (OEI) caracterizar aspectos voltados ao comportamento das pessoas, apenas três dimensões, são integradas nesse constructo: disposição individual de assumir riscos, ser inovador e proativo (Kollmann, Christofor, \& Kuckertz, 2007; Bolton, \& Lane, 2012; Goktan, \& Gupta, 2013).

\subsection{Orientação Empreendedora Individual voltada para o Social}

A abordagem integrativa dos aspectos afetivos e cognitivos faz muito sentido nos estudos sobre orientação empreendedora individual, principalmente, em se tratando do empreendedorismo social. Porque ao buscarem por soluções e atendimento a uma necessidade básica da sociedade em seus negócios, esses empreendedores mantém um comportamento cognitivo altamente influenciado pelos aspectos da afetividade, alavancando recursos que não controlam com um forte senso de responsabilidade (Bornstein \& Davis, 2010).

Alguns estudos já possibilitaram compreender o desempenho da intuição e da subjetividade nos processos de decisão empreendedora, principalmente, em relação à disposição para inovar. Os valores pessoais foram indicados por Krueger (2007) como determinantes no empreendedorismo porque as crenças profundas sustentam a criação de sentido, a tomada de decisão e o subsequente comportamento empreendedor. Kierkley (2016) descobriu que engajarse no empreendedorismo é uma forma de comportamento autodeterminado por valores motivacionais como independência, criatividade, ambição e ousadia.

Para muitos autores (Luthans, Youssef \&Avolio, 2007; Luthans \& Youssef, 2004), o capital psicológico traz parâmetros relacionados à pessoa do empreendedor - quem ele é, como se comporta e reage frente às adversidades em termos emocionais e comportamentais. A afetividade tem um sentido pleno: está relacionada às vivências e motivações das pessoas. E o cognitivo organiza o pensamento, ajuda a resolver problemas e buscar soluções. 
Os empreendedores sociais tentam criar e sustentar um valor como seus principais impulsionadores (Dees, 2001), mas com uma dificuldade tremenda: enquanto a proposição de valor nos mercados tradicionais pode fornecer vantagens substanciais para os investidores, a proposta de valor do empreendedor social tem como alvo uma população mal servida, negligenciada e que não tem meios financeiros ou influência política para alcançar o benefício transformador por conta própria (Martin \& Osberg, 2007). Desta forma, o empreendedor social deve estar disposto a assumir riscos da mesma forma que outros empreendedores estão, porém, seus drivers de decisão consideram elementos para além de seus interesses individuais e do negócio.

O comportamento inovador dos empreendedores sociais se volta à busca contínua de ideias para resolver problemas sociais e/ou ambientais. São transformadores do setor social porque atuam nas causas dos problemas e não nos sintomas a fim de criar mudanças e melhorias sustentáveis (Dees, 2001).

A proatividade social é observada nas práticas de vanguarda em querer tornar o mundo um lugar melhor. O capital psicológico do indivíduo pode influenciar essa questão. Os pesquisadores afirmam que os empreendedores são sujeitos suscetíveis a experimentar as emoções na tomada de decisão (Podoynitsyna, Van der Bij \& Song, 2012) e essas decisões podem estar relacionadas com reconhecimento, criação, avaliação e/ou exploração de possíveis oportunidades (Cardon et al., 2012). Os empreendedores sociais estão focados nas oportunidades que os problemas sociais podem trazer e, ao contrário do que se possa imaginar, não são motivados pela compaixão, mas pelo compromisso em obter resultados também para os seus empreendimentos, buscando eficiência nos processos e iniciativas, parcerias e colaborações (Dess, 2001).

$\mathrm{O}$ empreendedorismo vem sendo referenciado como um processo direcionado por valores (Morris \& Schindhutte, 2005), nos quais as crenças estão inseridas no comportamento e posicionam o indivíduo para a expressão empreendedora. Os valores raramente são aplicados de maneira consciente como busca de uma resposta a uma ação, mas podem ser ativados em situações que são percebidas como problemáticas (Schwartz, 2004). Então, se os valores influenciam como o indivíduo pensa, lideram preferências e influenciam percepções, interpretações, decisões e planejamento de ações concretas (Verplanken \& Holland, 2002; Feather, 1992; Shane, 2003; Schwartz \& Bardi, 2001), no empreendedorismo social devem ser o gatilho que movimenta os sujeitos aos negócios sociais e em ações para mudar o mundo, suas comunidades, seu país.

\section{Metodologia}

\subsection{Desenvolvimento da escala de OEI-Social}

Alguns instrumentos de mensuração da OEI são encontrados na literatura. Todos adaptaram os itens de observação da OE para avaliar os indivíduos (Kollmann, Stöckmann, Meves \& Kensbock, 2016; Bolton e Lane, 2012). Essas escalas vêm sendo usadas em diferentes pesquisas com satisfatório grau de mensuração (Kollmann, Stöckmann, Meves \& Kensbock, 2016; Qureshi \& Fawad Mahdi, 2014; Robinson, 2014; Robinson et al., 1991). 
Kraus et al. (2017) interessaram-se em mensurar a orientação empreendedora voltada para o social e, assim, desenvolveram uma escala que contempla as três dimensões consolidadas propostas por Miller (1983) e inspirada na escala de OE de Covin e Slevin (1991), adequando-os aos objetivos sociais da empresa: tomada de risco social; inovação social; proatividade social. Adicionam a sua escala, o fator Sociabilidade, que está relacionada à missão do negócio em servir a um propósito social antes do lucro. Os autores apresentam a validação de conteúdo dos itens, mas ainda não há nenhuma publicação de estudos sobre a validação da escala.

Seguimos a metodologia desses estudos anteriores que construíram escalas de orientação empreendedora individual inspiradas na literatura e nos instrumentos empíricos da OE e propomos a criação de uma escala de OEI-Social. Essa segunda parte do artigo apresenta as etapas iniciais de criação dessa escala, a saber: geração de itens e validação de face-conteúdo conforme Churchill (1979), DeVellis (2003) e Johnson \& Morgan (2016).

\subsection{Geração de itens}

Visto que a orientação empreendedora individual é uma variável latente e, portanto, não pode ser observada diretamente, seguimos os procedimentos de geração de itens com o propósito de capturar seu conteúdo relevante e observável (Johnson \& Morgan, 2016).

A revisão bibliográfica contribuiu para o melhor entendimento sobre o conceito de orientação empreendedora individual voltada para o empreendedorismo social. Ela envolve as três dimensões clássicas do empreendedorismo, adequadas ao contexto social, e mais uma variável que contemple a influência que os valores imprimem nesse comportamento. A proposição teórica que guia a construção dos itens de escala da OEI-Social considera que esse é um constructo de segunda ordem compreendido, portanto, por quatro dimensões: proatividade social, inovação social, tomada de risco social e uma quarta, relacionada às crenças, inclinação e interesse que direcionam os indivíduos às ações relativas à transformação do mundo. Trataremos dessa quarta dimensão à frente, visto que optamos pela inserção de uma escala já validada que não passará pelo teste de validação de conteúdo. A Figura 1 apresenta as proposições teóricas para cada dimensão que será avaliada nesta etapa do estudo.

\begin{tabular}{|c|l|l|}
\hline $\mathrm{P}_{1}$ & $\begin{array}{l}\text { Proatividade } \\
\text { Social }\end{array}$ & $\begin{array}{l}\text { A proatividade social refere-se à capacidade e à disposição de um indivíduo em realizar } \\
\text { ações para efetuar mudanças no ambiente orientadas a objetivos sociais/ambientais e a } \\
\text { busca antecipada por novas soluções/oportunidades. }\end{array}$ \\
\hline $\mathrm{P}_{2}$ & $\begin{array}{l}\text { Inovação } \\
\text { Social }\end{array}$ & $\begin{array}{l}\text { Predisposição para identificar e avaliar oportunidades sociais e ambientais, bem como, o } \\
\text { desejo de introduzir novidades por meio da experimentação e de processos criativos de } \\
\text { modo a criar novos produtos e serviços de soluções para o social e/ou ambiental. }\end{array}$ \\
\hline $\mathrm{P}_{3}$ & $\begin{array}{l}\text { Tomada de } \\
\text { Risco Social }\end{array}$ & $\begin{array}{l}\text { Assumir riscos sociais está relacionado à disposição em comprometer recursos a novos } \\
\text { projetos sociais/ambientais para o desenvolvimento de soluções/oportunidades. }\end{array}$ \\
\hline
\end{tabular}

Figura 1: Proposição teórica das dimensões escala de OEI-SOCIAL

Fonte: Autores, 2019.

Como havia poucas referências sobre o construto OEI-Social na literatura, seguimos uma abordagem dedutiva e indutiva para obter nossa lista de itens inicial. Os itens foram gerados com base na revisão de literatura relacionada ao conceito de empreendedorismo 
social e orientação empreendedora individual. Também, nas escalas de OEI (Bolton \& Lane, 2012) e de OE Social (Kraus et al., 2017). Foram criadas 24 assertivas (Figura 2), adequadas às dimensões do constructo orientação empreendedora individual voltada ao social (proatividade social, inovação social, tomada de risco social).

\begin{tabular}{|c|c|}
\hline Proa-S-1 & $\begin{array}{l}\text { Eu costumo organizar e liderar ações de ajuda social e/ou ambiental, mesmo antes de alguém me } \\
\text { pedir. }\end{array}$ \\
\hline Proa-S-2 & Eu crio projetos para ajudar as pessoas e/ou a natureza, mesmo que ninguém esteja fazendo isso. \\
\hline Proa-S-3 & $\begin{array}{l}\text { Eu prefiro "acelerar" e fazer as coisas para tornar o mundo um lugar melhor em vez de sentar e } \\
\text { esperar que alguém faça isso. }\end{array}$ \\
\hline Proa-S-4 & $\begin{array}{l}\text { Eu busco transformar a realidade social e/ou ambiental da minha cidade (bairro), mesmo que } \\
\text { ninguém esteja fazendo isso. }\end{array}$ \\
\hline Proa-S-5 & $\begin{array}{l}\text { Eu faço ações para transformar o mundo em um lugar melhor para viver. Mesmo que ninguém } \\
\text { esteja fazendo isso. }\end{array}$ \\
\hline Proa-S-6 & Eu crio projetos sociais e/ou ambientais e convido meus amigos e/ou desconhecidos a participarem. \\
\hline Risc-S-1 & Eu não tenho medo de liderar uma organização sem fins lucrativos. \\
\hline Risc-S-2 & criaria uma empresa voltada para resolver problemas sociais e/ou ambientais. \\
\hline Risc-S-3 & Eu investiria tempo e dinheiro em negócios que envolvem soluções para o social e/ou ambiental. \\
\hline Risc-S-4 & $\begin{array}{l}\text { Estou disposto a investir tempo e dinheiro em algo que possa transformar o mundo em um lugar } \\
\text { melhor para se viver. }\end{array}$ \\
\hline Risc-S-5 & Eu gostaria de me aventurar em projetos para solucionar problemas sociais e/ou ambientais. \\
\hline Risc-S-6 & a te \\
\hline Risc-S-7 & $\begin{array}{l}\text { Ações corajosas são necessárias para atingir resultados na solução de problemas sociais e/ou } \\
\text { ambientais. }\end{array}$ \\
\hline Risc-S-8 & iito que ações ousadas podem transformar o mundo em um lugar melhor para se viver. \\
\hline Risc-S-9 & rganização não governamental. \\
\hline Inov-S-1 & mbientais. \\
\hline Inov-S -2 & $\begin{array}{l}\text { Acredito que a transformação do mundo passa por novas ideias para velhos problemas sociais e } \\
\text { ambientais. }\end{array}$ \\
\hline Inov-S-3 & Eu gosto de propor projetos incomuns para solucionar problemas ações sociais e ambientais. \\
\hline Inov-S-4 & $\begin{array}{l}\text { Sou a favor de experimentar abordagens originais para a solução de problemas sociais e ambientais, } \\
\text { em vez de usar métodos que os outros já usam. }\end{array}$ \\
\hline Inov-S-5 & $\begin{array}{l}\text { Eu sou uma pessoa determinada na busca de soluções inovadoras para os problemas sociais e } \\
\text { ambientais. }\end{array}$ \\
\hline Inov-S-6 & $\begin{array}{l}\text { Normalmente, minhas ideias para projetos sociais e/ou ambientais trazem soluções que ninguém } \\
\text { tinha pensado antes. }\end{array}$ \\
\hline Inov-S-7 & $\begin{array}{l}\text { Eu me preocupo em propor novas soluções para problemas sociais e ambientais. Algo que ninguém } \\
\text { nunca fez antes. }\end{array}$ \\
\hline Inov-S-8 & $\begin{array}{l}\text { Em geral, eu prefiro buscar por soluções únicas para projetos sociais e ambientais em vez de adotar } \\
\text { ideias já testadas. }\end{array}$ \\
\hline Inov-S-9 & $\begin{array}{l}\text { Eu acredito que sou uma pessoa ágil e criativa na busca de novas soluções para problemas sociais e } \\
\text { ambientais. }\end{array}$ \\
\hline
\end{tabular}

Figura 2: Itens de observação para a escala OEI-Social

Fonte: Autores, 2019

\subsection{Validação de conteúdo}

Uma obrigação metodológica ao desenvolvimento de escalas envolve a avaliação da validade de conteúdo dos itens observáveis constituintes das variáveis (Churchill, 1979; 
DeVellis, 2003). Nesta etapa, os itens de uma escala são apresentados a especialistas, a fim de avaliarem sua adequação ao construto que se pretende medir.

Participaram dessa etapa, 24 pesquisadores doutores em Empreendedorismo de universidades públicas e privadas de referência no Brasil. Eles receberam um link de acesso ao conjunto de itens e às explicações de cada dimensão. Após a leitura, eles deveriam indicar a qual dimensão cada frase pertenceria. A fim de evitar o cansaço, o desinteresse, o vício no olhar e as respostas automáticas, a listagem de itens receberam o tratamento de randomização completa, por itens e por respostas. Ou seja, as perguntas foram embaralhadas a fim de capturar a atenção dos respondentes. Esse instrumento foi alocado no site de questionários SurveyMonkey ${ }^{\mathrm{R}}$.

A análise das respostas dos especialistas demostrou que algumas frases eram confusas, com itens sendo classificados em até três dimensões diferentes ou sendo interpretados em dimensões não previstas pela teoria.

Todas nesta situação foram eliminadas da lista. Percebe-se que a dimensão que teve maior perda de afirmativas foi tomada de risco. De nove afirmativas iniciais, apenas 04 foram validadas. Apenas os itens que tiveram índice de menção nas dimensões corretas acima de $50 \%$ foram selecionados para seguirem na escala. DeVellis (2003) afirma que esse é o percentual mínimo para que os itens sejam considerados representativos de uma dimensão. Apenas uma frase obteve 52\%. Todos os outros ficaram acima de $60 \%$, conferindo qualidade aos itens. Vale destacar que das 24 afirmações iniciais, 18 foram validadas pelos especialistas, confirmando a qualidade das proposições teóricas para a geração de itens. E, portanto, a etapa de validação de conteúdo foi finalizada em apenas uma rodada. A Tabela 1 apresenta o resultado da validação de conteúdo com destaque aos itens que se mostraram mais ajustados às dimensões e, portanto, foram validados a constituírem a escala de OEI-Social.

\subsection{A quarta dimensão - mudança mundial}

A análise da literatura indica o papel de importância que os elementos da afetividade imprimem ao comportamento empreendedor social. Assim, é possível propor que, da mesma forma que Sociabilidade representa a maneira como a empresa executa o seu negócio social e, assim, configura-se como uma quarta dimensão na escala de OE-Social de Kraus et al. (2017), a quarta dimensão da escala de OEI-Social deve ser uma que represente as orientações que iniciam, guiam e mantém uma ação pró-social dos empreendedores sociais.

Buscou-se, portanto, na literatura, uma escala que representasse esse comportamento pró-social. Encontramos a World Change Scale (WCS), de Oceja e Salgado (2013). Esse instrumento atua fazendo uma associação entre uma situação e os cinco itens formativos da escala referentes às mudanças positivas no mundo. $\mathrm{O}$ questionário original pede aos entrevistados que avaliem a ação de ajuda por meio de uma escala Likert de 7 pontos $(1=$ concordo totalmente, $7=$ discordo totalmente). De acordo com seus autores, em teste de validação da escala, a WCS mostrou-se adequada a medir a orientação e motivação de um indivíduo em agir pró-socialmente em função de tornar o mundo um lugar melhor.

Realizamos a tradução e a adaptação dos cinco itens da WCS ao contexto geral de empreendedorismo social. A Figura 3 apresenta os itens originais da escala, traduzidos e 
adaptados ao propósito da escala de OEI-Social. Na adaptação e tradução, chamaremos essa escala de Mudança Social.

\begin{tabular}{|l|l|}
\hline \multicolumn{1}{|c|}{ Itens originais WCS } & \multicolumn{1}{c|}{ Itens adaptados Mudança Social } \\
\hline These kinds of things can also change the world. & $\begin{array}{l}\text { Eu acredito que ações sociais e ambientais também podem } \\
\text { mudar o mundo. }\end{array}$ \\
\hline $\begin{array}{l}\text { Humanity and its environment depend on our } \\
\text { actions. }\end{array}$ & $\begin{array}{l}\text { Eu acredito que a humanidade e o meio-ambiente } \\
\text { dependem de nossas ações. }\end{array}$ \\
\hline The benefits go far beyond the simple helping act. & $\begin{array}{l}\text { Eu acredito que os benefícios de ações sociais e ambientais } \\
\text { vão muito além do simples ato de ajudar as pessoas e a } \\
\text { Natureza. }\end{array}$ \\
\hline Our decisions can achieve huge changes & $\begin{array}{l}\text { Eu acredito que nossas decisões podem realizar grandes } \\
\text { mudanças. }\end{array}$ \\
\hline It is a way to make the world a better place & $\begin{array}{l}\text { Eu acredito que desenvolver ações em prol das pessoas e } \\
\text { do meio ambiente é uma maneira de tornar o mundo um } \\
\text { lugar melhor. }\end{array}$ \\
\hline
\end{tabular}

\section{Figura 3: Escala Mudança Social}

Fonte: Oceja et al. (2013), adaptada pelos autores (2019)

Como o grau de envolvimento dos sujeitos em ações pró-sociais é previsto pelo WCS quando a situação é definida em termos de melhoria do bem-estar de uma situação concreta, a quarta proposição teórica desse estudo considera ser a inserção da escala de WCS adequada ao instrumento de avaliação e mensuração da OEI-S para representar o comportamento afetivo do empreendedor social frente aos seus negócios. Assim:

$\mathrm{P}_{4}$ : A escala World Change Scale (Oceja \& Salgado, 2013) é uma dimensão da escala de OEI-S, servindo para medir o comportamento pró-social dos empreendedores sociais.

\section{Discussões}

A compreensão da OEI voltada para o Social como um comportamento empreendedor identificado com questões sociais $\mathrm{e}$ ambientais retoma $\mathrm{o}$ pilar fundamental do empreendedorismo: da destruição inovadora e criativa do capitalismo (Schumpeter, 1928). A escala aqui proposta tem o objetivo de avaliar esse comportamento disruptivo.

A escala OEI-Social é muldimensional e intenta avaliar e mensurar o comportamento afetivo e cognitivo do empreendedor social representado pelas quatro dimensões justificadas na revisão bibliográfica sobre o fenômeno.

Os diferentes conceitos de negócios sociais apresentados pela literatura permitem-nos identificar uma lógica de mercado predominantemente social. Por isso, avaliar os comportamentos de proatividade, inovação e de tomada de risco desses empreendedores requer considerar o elemento social e as normas coletivas que regem o continuum (Austin, 2002) dessas organizações, sejam elas inseridas na economia solidária ou sejam empresas de impacto socioambiental. Os itens de observação dessas três dimensões validados nesse estudo pretendem capturar essas conexões. 


\section{Tabela 1: Validação de Conteúdo}

\begin{tabular}{|c|c|c|c|}
\hline & $\begin{array}{l}\text { Proatividade } \\
\text { Social }\end{array}$ & $\begin{array}{l}\text { Inovação } \\
\text { Social }\end{array}$ & $\begin{array}{l}\text { Tomada de } \\
\text { Risco }\end{array}$ \\
\hline $\begin{array}{l}\text { Eu costumo organizar e liderar ações de ajuda social e/ou ambiental, mesmo antes de } \\
\text { alguém me pedir. }\end{array}$ & $100 \%$ & $0 \%$ & $0 \%$ \\
\hline $\begin{array}{l}\text { Eu crio projetos para ajudar as pessoas e/ou a natureza, mesmo que ninguém esteja } \\
\text { fazendo isso. }\end{array}$ & $61 \%$ & $26 \%$ & $13 \%$ \\
\hline $\begin{array}{l}\text { Eu busco transformar a realidade social e/ou ambiental da minha cidade (bairro), } \\
\text { mesmo que ninguém esteja fazendo isso. }\end{array}$ & $74 \%$ & $22 \%$ & $4 \%$ \\
\hline $\begin{array}{l}\text { Eu faço ações para transformar o mundo em um lugar melhor para viver. Mesmo } \\
\text { que ninguém esteja fazendo isso. }\end{array}$ & $96 \%$ & $4 \%$ & 0 \\
\hline $\begin{array}{l}\text { Eu prefiro "acelerar" e fazer as coisas para tornar o mundo um lugar melhor em vez } \\
\text { de sentar e esperar que alguém faça isso. }\end{array}$ & $83 \%$ & $13 \%$ & $4 \%$ \\
\hline $\begin{array}{l}\text { Eu crio projetos sociais e/ou ambientais e convido meus amigos e/ou desconhecidos a } \\
\text { participarem. }\end{array}$ & $96 \%$ & $4 \%$ & 0 \\
\hline Eu me interesso por novas respostas para problemas sociais e ambientais. & $35 \%$ & $65 \%$ & 0 \\
\hline $\begin{array}{l}\text { Acredito que a transformação do mundo passa por novas ideias para velhos } \\
\text { problemas sociais e ambientais. }\end{array}$ & $4 \%$ & $96 \%$ & 0 \\
\hline $\begin{array}{l}\text { Eu gosto de propor projetos incomuns para solucionar problemas ações sociais e } \\
\text { ambientais. }\end{array}$ & $22 \%$ & $70 \%$ & $8 \%$ \\
\hline $\begin{array}{l}\text { Sou a favor de experimentar abordagens originais para a solução de problemas } \\
\text { sociais e ambientais, em vez de usar métodos que os outros já usam. }\end{array}$ & $4 \%$ & $79 \%$ & $17 \%$ \\
\hline $\begin{array}{l}\text { Eu sou uma pessoa determinada na busca de soluções inovadoras para os problemas } \\
\text { sociais e ambientais. }\end{array}$ & $35 \%$ & $65 \%$ & 0 \\
\hline $\begin{array}{l}\text { Normalmente, minhas ideias para projetos sociais e/ou ambientais trazem soluções } \\
\text { que ninguém tinha pensado antes. }\end{array}$ & $4 \%$ & $83 \%$ & $13 \%$ \\
\hline $\begin{array}{l}\text { Eu me preocupo em propor novas soluções para problemas sociais e ambientais. } \\
\text { Algo que ninguém nunca fez antes. }\end{array}$ & $9 \%$ & $87 \%$ & $4 \%$ \\
\hline $\begin{array}{l}\text { Em geral, eu prefiro buscar por soluções únicas para projetos sociais e ambientais em } \\
\text { vez de adotar ideias já testadas. }\end{array}$ & $9 \%$ & $78 \%$ & $13 \%$ \\
\hline Eu criaria uma empresa voltada para resolver problemas sociais e/ou ambientais. & $26 \%$ & $22 \%$ & $52 \%$ \\
\hline Eu investiria tempo e dinheiro para criar uma organização não governamental. & $13 \%$ & $4 \%$ & $83 \%$ \\
\hline $\begin{array}{l}\text { Estou disposto a investir tempo e dinheiro em algo que possa transformar o mundo } \\
\text { em um lugar melhor para se viver. }\end{array}$ & $22 \%$ & $13 \%$ & $65 \%$ \\
\hline $\begin{array}{l}\text { Eu investiria tempo e dinheiro em negócios que envolvem soluções para o social e/ou } \\
\text { ambiental. }\end{array}$ & $26 \%$ & $8 \%$ & $65 \%$ \\
\hline $\begin{array}{l}\text { Eu gostaria de me aventurar em projetos para solucionar problemas sociais e/ou } \\
\text { ambientais. }\end{array}$ & $30 \%$ & $31 \%$ & $39 \%$ \\
\hline $\begin{array}{l}\text { Ações corajosas são necessárias para atingir resultados na solução de problemas } \\
\text { sociais e/ou ambientais. }\end{array}$ & $48 \%$ & $4 \%$ & $48 \%$ \\
\hline Eu não tenho medo de liderar uma organização sem fins lucrativos. ${ }^{\mathbf{e}}$ & $17 \%$ & $83 \%$ & 0 \\
\hline Não tenho medo de direcionar minha carreira para a área social ou ambiental. ${ }^{\mathbf{e}}$ & $13 \%$ & $87 \%$ & 0 \\
\hline $\begin{array}{l}\text { Eu acredito que ações ousadas podem transformar o mundo em um lugar melhor } \\
\text { para se viver. }\end{array}$ & $30 \%$ & $44 \%$ & $26 \%$ \\
\hline $\begin{array}{l}\text { Eu acredito que sou uma pessoa ágil e criativa na busca de novas soluções para } \\
\text { problemas sociais e ambientais. }\end{array}$ & $43 \%$ & $48 \%$ & $9 \%$ \\
\hline
\end{tabular}

Fonte: Autores, 2019

Nota: Os itens ${ }^{\mathbf{e}}$ não foram validados. 
A dimensão tomada de risco social associa-se aos riscos substanciais que o empreendedor está disposto a assumir para servir ao propósito social de fornecer soluções e atendimento a alguma necessidade básica da sociedade.

A inovação social representa o comportamento contínuo na busca de ideias para resolver problemas sociais por meio de produtos e serviços. Barki et al. (2015) afirmam que o empreendedor social busca inovação social para melhorar o mundo e não mede esforços para mobilizar recursos necessários para a promoção de mudanças sociais (Elkington \& Hartigan, 2008).

Os itens de proatividade social buscam capturar as iniciativas relacionadas à busca antecipada de oportunidades para oferecer à sociedade soluções para a melhoria da vida das pessoas. Ela é crucial no empreendedorismo social porque tem a capacidade de vislumbrar caminhos, ideias e soluções que, acompanhadas por atividades inovativas, constituem um negócio social.

Já a dimensão Mudança Social reflete o entendimento que o empreendedorismo social atua inserido em uma rede fundamentada em princípios coletivos, de reciprocidade e confiança mútua. Essa dimensão foi incluída na escala de OEI-Social para retratar a proposição que o comportamento de criar impacto social e ou ambiental positivo deve ser intencional, motivador e contínuo nos empreendedores sociais. É, assim, um comportamento pró-social.

A OEI-Social está relacionada às normas e valores sociais que criam relacionamentos e consolidam ações que afetam os resultados econômicos tradicionais de maneira importante. Assim, compreendê-la, avaliá-la e mensurá-la por meio de um instrumento empírico adequado permite direcionar os sujeitos a atuarem em projetos empresariais direcionados a um equilíbrio entre o sucesso material, o atendimento dos valores pessoais e do bem-estar da sociedade.

$O$ resultado dessa pesquisa evidencia, portanto, que a OEI inserida no empreendedorismo social tem aspectos comportamentais próprios e específicos e, portanto, amplia o conceito original de orientação empreendedora individual - OEI.

\section{Considerações finais}

A revisão teórica sobre o tema indica que o empreendedorismo social carrega um alto potencial de transformação social e econômica. Não apenas na geração de trabalho e renda e na arrecadação de impostos, mas também na transformação de uma realidade de conquista de cidadania e empoderamento das comunidades (Barki et al., 2013). O Mapa das Organizações da Sociedade Civil, do Ipea, indica que existem 400 mil OSCs em atuação no Brasil (Ipea, 2015), mas esses números são referentes apenas às organizações pertencentes ao terceiro setor. A Pipe Social (2019) mapeou 1002 negócios de impacto socioambiental em todas as regiões do país.

Mas, ainda são incipientes as pesquisas sobre o comportamento empreendedor social. O levantamento das publicações sobre o tema que realizamos nas principais bases de pesquisa internacionais (EBSCO, PROQUEST, GOOGLE SCHOLAR) e nacionais (CAPES; SCIELO; SPELL), voltados para a orientação empreendedora individual sob os termos "individual entrepreneurial orientation", "social individual entrepreneurial orientation, "orientação 
empreendedora individual" e "orientação individual empreendedora ao empreendedorismo social" (por recência e relevância) retornou um baixo número de incidências.

Assim, o campo de investigação à OEI-Social é vasto e se abre a diferentes indagações e contribuições, tanto à teoria como aos negócios sociais, fundamentalmente, devido à relevância das dimensões comportamentais desse constructo à competitividade.

Adiante destas constatações, esse estudo assumiu o desafio de entender, avaliar e mensurar o comportamento afetivo e cognitivo dos indivíduos que empreendem em negócios sociais por meio de uma escala, aqui nominada Escala de OEI-Social. Ele contribui, não apenas no âmbito empírico das pesquisas, mas também à teoria, em propor o comportamento pró-social como objeto de análise. Esse trabalho não termina aqui e deve prosseguir para o teste de avaliação do poder de mensuração do instrumento.

Ele também permite propor outros estudos em direção ao maior entendimento sobre a relação entre a influência dos valores sociais e o comportamento do empreendedor social, bem como, avançar nas reflexões e análises inserindo esse fenômeno nas teorias econômicas.

A sua relevância não se pauta apenas pelos achados teóricos e empíricos. Mas, também, ao estímulo à aproximação entre a Academia e os agentes sociais inseridos e responsáveis por essas organizações. Essa integração - pesquisa e mercado - é fundamental para alicerçar de maneira mais consistente, os caminhos para a disrupção de uma economia que segrega e individualiza em direção a uma economia recíproca e altruísta que promova o desenvolvimento econômico de comunidades e do país.

\section{Referências}

Austin, J. (2002). The collaboration challenge: how nonprofits and businesses succeed through strategic alliances. San Francisco, CA: Jossey-Bass.

Austin, J.; Stevenson, H. \& Wei-Skillern, J. (2012). Social and Commercial Entrepreneurship: Same, Different, or Both? Revista da Administração. 47 (3), 370-384.

Barki, E., Izzo, D., Torres, H., \& Aguiar, L. (Orgs) (2013). Negócios com impacto social no Brasil. São Paulo: ed. Petrópolis, p.256.

Barki, E., Comini, G., Cunliffe, A., Hart, S. L., \& Rai, S. (2015). Social en trepreneurship and social business: Retrospective and prospective research. RAE-Revista de Administração de Empresas, 55(4), 380-384. doi:10.1590/S0034-759020150402

Bolton, D.L. \& Lane, M.D. (2012). Individual entrepreneurial orientation: development of a measurement instrument. Education + Training. 54 (2/3), 219-233.

Bornstein, D., \& Davis, S. (2010). Social entrepreneurship: what everyone needs to know. New York, NY: Oxford University Press, 147 p.

Borzaga, C., Depedri, S., \& Galera, G. (2012). Interpreting social enterprises. Rev. Adm. (São Paulo) [online]. V..47, n.3, pp.398-409. 
Cardon, M. S., Zietsma, C., Saparito, P., Matherne, B. P., \& Davis, C. (2012) A tale of passion: new insights into entrepreneurship from a parenthood metaphor. Journal of Business Venturing, 20, pp. 2345 .

Churchill, G.A. (1979), A paradigm for developing better measures of marketing constructs. Journal of Marketing Research, 16 (1), 64-73.

Covin, J. G, \& Slevin, DP. (1991). A conceptual model of entrepreneurship as firm behavior. Entrepreneurship: Theory \& Practice. 16, 7-25.

Dees, J.G. (2001). The meanings of 'social entrepreneurship, working paper. Stanford University, Stanford, CA.

DeGennaro, M. P., Wright, C. W., \& Panza, N. R. (2016). Measuring Entrepreneurial Orientation in an assessment center: An individual level-of-analysis study. The Psychologist-Manager Journal, 19(1), 1.

Deloitte. The Deloitte Millennial Survey 2018. Disponível em: https://www2.deloitte.com/global/en/pages/about-deloitte/articles/millennialsurvey.html.

DeVellis, R.F. (2003). Scale Development: theory and applications. Thousand Oaks, Sage Publications

Feather, N.T. (1992). Values, valences, expectations and actions. Journal of Science Issues. 48, 109 124.

Godói-de-Sousa, E. \& Fischer, R. M. (2012). The succession process at social enterprises in Brazil. Revista da Administração. 47(3).

Godói-de-Sousa, E. (2010). O processo sucessório em associações produtivas no Brasil: estrutura, desafios e oportunidades. São Paulo. Tese (Doutorado em Administração). FEA-Universidade de São Paulo.

Goktan, A. B., \& Gupta, V. K. (2015). Sex, gender, and individual entrepreneurial orientation: evidence from four countries. International Entrepreneurship and Management Journal, 11(1), 95112.

Johnson, R.L., Morgan, G.B. (2016). Survey Scales: A Guide to Development, Analysis, and Reporting,1st ed. The Guilford Press, New York, NY

Kierkley, W.W.(2016). Entrepreneurial behaviour: the role of values. International Journal Of Entrepreneurial Behavior \& Research. 22 (3)

Kollmann, T., Christofor, J., \& Kuckertz, A.(2007). Explaining Individual Entrepreneurial Orientation: Conceptualization of a Cross-Cultural Research Framework, International Journal of Entrepreneurship and Small Business 4(3):325-340. 
Kollmann, T., Stöckmann, C., Meves, Y., \& Kensbock, J. (2016). When members of entrepreneurial teams differ linking diversity in individual-level entrepreneurial orientation to team performance. Small Bus Econ. Springer Science Business Media. New York.

Kraus, S. et al. (2017). Social entrepreneurship orientation: development of a measurement scale. International Journal of Entrepreneurial Behaviour and Research.1-34.

Krueger, N.F. (2007), What lies beneath? The experiential essence of entrepreneurial thinking. Entrepreneurship Theory \& Practice, 31(1), 123-138.

IPEA. Mapa das Organizações da Sociedade Civil. In: https://mapaosc.ipea.gov.br/dadosindicadores.html.

Martin, R. L., \& Osberg, S. (2007. Social entrepreneurship: The case for defnition. Stanford Social Innovation Review, 5(2), 28-39.

Miller, D. (1983). The Correlates of Entrepreneurship in Three Types of Firms. Management Science 29 (7), 770-791.

Morris, M. Schindhutte, M. (2005). Entrepreneurial values and the ethnic enterprises: an examination of six subculture. Journal of Small l Business Management. 43 (4), 453-479

Oceja, L., \& Salgado, S. (2012). Why do we help? World change orientation as an antecedent of prosocial action. European Journal of Social Psychology, 43(2), 127-136.

Padilla-Meléndez, A., Fernández-Gámez, M. A., \& Molina-Gómez, J. (2014). Feeling the risks: effects of the development of emotional competences with outdoor training on the entrepreneurial intent of university students. International Entrepreneurship and Management Journal, 10(4), 861-884.

Pipe Social. Mapa de Negócios de Impacto Social + Ambiental. In: https://pipe.social/mapa2019.

Prahalad, C. K. (2005). Riqueza na Base da Pirâmide. Editora Bookman.

Podoynitsyna, K.; Van der Bij, H.; Song, M. (2012). The Role of Mixed Emotions in the Risk Perception of Novice and Serial Entrepreneurs. Entrepreneurship theory and practice. 36 (1), 115140.

Quintão, C. (2004). Empreendedorismo social e oportunidades de construção do próprio emprego. In: Seminário Trabalho Social E Mercado De Emprego. Porto. Disponível em: <http://www.letras.up.pt/isociologia/uploads/files/Working4.pdf>.

Qureshi, M.S. \& Mahdi, F. (2014). Impact of effectuation based interventions on the intentions to start a business. Business Review, 9 (2).

Reficco, E., Gutiérrez, R., \& Trujillo, D. (2006). Empresas sociales: ¿una especie en busca de reconocimiento? R.Adm., São Paulo, v.41, n.4, p.404-418, 
Robinson, S. (2014). Elements of Entrepreneurial orientation and their relationship to entrepreneurial intention. Journal of Entrepreneurship Education, 17 (2),

Robinson, P., \& Huefner, J. (1991). An attitude approach to the prediction of entrepreneurship. Entrepreneurship Theory \& Practice. Summer, 15(4), 13 - 30.

Schumpeter, Joseph. (1928).The instability of capitalism. The Economic Journal. 361-386.

Schwartz, S. H. (2004). Mapping and interpreting cultural differences around the world . In Vinken, H. Soeter, J. and Ester, P. (eds.) Comparing cultures, dimensions of culture in a comparative perspective, Brill: Leiden, The Netherlands,1-65

Schwartz, S. H., \& Bardi, A. (2001). Value hierarchies across cultures: Taking a similarities perspective. Journal of Cross-Cultural Psychology. 32, 268-290.

Soeter, J. \& Ester, P. (eds.) Comparing cultures, dimensions of culture in a comparative perspective, Brill: Leiden, The Netherlands,1-65.

Shane, S. (2003). A general theory of entrepreneurship; the individual opportunity nexus. Edward Elgar Publising Limited. Cheltenham, UK.

Teodosio, A. S. S., \& Comini, G. (2012) Negócios inclusivos e pobreza: perspectivas no contexto brasileiro. Rev. Adm. (São Paulo) [online]. V.47, n.3, pp.410-421.

Verplanken, B; Holland, R.W. (2002) Motivated decision making: effects of activation and selfcentrality of values on choices and behavior. Jornal of Personal and Social Psychology, 82, 43447.

Wiklund, J.\& Shepherd, D. (2005) Entrepreneurial orientation and small business performance: a configurational approach. Journal of Business Venturing, v. 20, n. 1, p. 71-91.

Yunus, M.; Moingeon, B.\& Lehmann-Ortega, L. (2010). Building Social Business Models: Lessons from the Grameen Experience. Long Range Planning. 43, 308-325.

Zadek, S.\& Thake, S. (1997). Send in the social entrepreneurs. New Statesman. (26) 31.

Zahra, S. A. (1993). A conceptual model of entrepreneurship as firm behaviour: a critique and extension. Entrepreneurship: Theory \& Practice, v. 16, n. 4, p. 5-21.

Zahra, S. A., \& Covin, J. G. (1995). Contextual influences on the corporate entrepreneurship performance relationship: a longitudinal analysis. Journal of Business Venturing, v. 10, n. 1, p. 43-58, January. 